OPEN ACCESS

Edited by:

Jaime Ribeiro-Filho,

Oswaldo Cruz Foundation (FIOCRUZ),

Brazil

Reviewed by:

Irwin Rose Alencar De Menezes,

Regional University of Cariri, Brazil

Sanguine Byun,

Yonsei University, South Korea

*Correspondence:

Ruoruo Zhang

465125310@qq.com

Xiufeng Zhang

zxf96178@126.com

Specialty section:

This article was submitted to Inflammation Pharmacology,

a section of the journal

Frontiers in Pharmacology

Received: 19 November 2021

Accepted: 15 December 2021

Published: 12 January 2022

Citation:

Shi J, Xia Y, Wang H, Yi Z, Zhang R and Zhang $X$ (2022) Piperlongumine Is an NLRP3 Inhibitor With Antiinflammatory Activity.

Front. Pharmacol. 12:818326. doi: 10.3389/fphar.2021.818326

\section{Piperlongumine Is an NLRP3 Inhibitor With Anti-inflammatory Activity}

\author{
Jie Shi ${ }^{1,2}$, Yang Xia ${ }^{1}$, Huihong Wang ${ }^{1}$, Zhongjie Yi $^{3}$, Ruoruo Zhang $^{4 *}$ and Xiufeng Zhang ${ }^{1 *}$ \\ ${ }^{1}$ Department of Respiratory Medicine, Second Affiliated Hospital of Hainan Medical University, Haikou, China, ${ }^{2}$ Department of \\ General Surgery, The Third Xiangya Hospital of Central South University, Changsha, China, ${ }^{3}$ Department of Plastic and Aesthetic \\ (Burn) Surgery, The Second Xiangya Hospital of Central South University, Changsha, China, ${ }^{4}$ Institute of Transplantation \\ Medicine, Second Affiliated Hospital of Hainan Medical University, Haikou, China
}

Piperlongumine $(\mathrm{PL})$ is an alkaloid from Piper longum $\mathrm{L}$. with anti-inflammatory and antitumor properties. Numerous studies have focused on its antitumor effect. However, the underlying mechanisms of its anti-inflammation remain elusive. In this study, we have found that PL is a natural inhibitor of Nod-like receptor family pyrin domain-containing protein-3 (NLRP3) inflammasome, an intracellular multi-protein complex that orchestrates host immune responses to infections or sterile inflammations. PL blocks NLRP3 activity by disrupting the assembly of NLRP3 inflammasome including the association between NLRP3 and NEK7 and subsequent NLRP3 oligomerization. Furthermore, PL suppressed lipopolysaccharide-induced endotoxemia and MSU-induced peritonitis in vivo, which are NLRP3-dependent inflammation. Thus, our study identified PL as an inhibitor of NLRP3 inflammasome and indicated the potential application of PL in NLRP3-relevant diseases.

Keywords: piperlongumine, NLRP3, Nek7, inflammasome assembly, inflammation

\section{INTRODUCTION}

Piperlongumine (PL) is a natural product from the fruit of long pepper and a form of traditional Chinese medicine (Wang et al., 2014). PL exhibits antitumor properties in serials of tumors including sarcoma, melanoma, gastrointestinal cancers, and bladder cancers by induction of autophagy, apoptosis, and cell cycle arrest through modulating ROS production (Chen et al., 2019; Rawat et al., 2020; Shin et al., 2020). Recent studies have found that PL shows potent anti-inflammatory effects in ovalbumin-induced asthma and airway inflammation, neuroinflammation, and psoriasis-like skin inflammation (Gu et al., 2018; Kim et al., 2018; Lu et al., 2019). However, the underlying mechanisms for PL anti-inflammation were all attributed to the NF- $\mathrm{kB}$ signal inhibition. Given the broad antiinflammatory effects of PL, we speculated that there still exists an unknown mechanism for PL in suppressing inflammatory responses.

The NLRP3 inflammasome is an intracellular multiprotein complex that is critical in protecting the host from infections or sterile injuries (Mao et al., 2013). NLRP3 can sense diverse stimuli including pathogen components, environment irritants, and host danger effectors, so its aberrant activation leads to many inflammatory diseases, such as sepsis (Mao et al., 2013), gout (Martinon et al., 2006), type 2 diabetes (Masters et al., 2010), atherosclerosis (Duewell et al., 2010; Bai et al., 2021), and Alzheimer's disease (Heneka et al., 2013). It consists of a sensor, a nucleotide-binding domain, a leucine-rich repeat, pyrin domain-containing protein 3 (NLRP3), an adaptor, the apoptosis-associated speck-like protein containing a CARD (ASC), and an effector, caspase-1 (Swanson et al., 2019). NLRP3 inflammasome activation is a two-step process. First, it needs a priming signal to upregulate the expression of NLRP3 and pro-IL- $1 \beta$, and the priming signal can be 
induced by various pathogen-associated molecular patterns (PAMPs) or through cytokines such as the tumor necrosis factor (TNF). Second, the inflammasome is formed and fully activated, which can be triggered by a wide variety of stimuli. Oligomerized NLRP3 recruits ASC and then forms a large complex to activate caspase-1, which induces the maturation of IL- $1 \beta$ and IL-18 as well as gasdermin D-mediated pyroptotic cell death.

In this study, we found that PL could inhibit the NLRP3 inflammasome activation in murine and human macrophages. Moreover, PL alleviated the lipopolysaccharide (LPS)-induced endotoxemia and MSU-induced peritonitis in vivo, which are NLRP3-dependent inflammations. Mechanistically, PL blocks NLRP3 inflammasome assembly by interrupting the interaction between NLRP3 and NEK7 and subsequent aggregation of NLRP3. Thus, our study identified PL as an NLRP3 inhibitor and indicated the potential application of PL in NLRP3-relevant diseases.

\section{MATERIALS AND METHODS}

\section{Animals}

Wild-type (WT) C57BL/6 mice (8-10 weeks old, weight between 20-25 g) were bought from Hunan SJA Laboratory Animal Co., Ltd. (Changsha, China) and were kept under SPF conditions with standard chows and a 12- h light/dark cycle. All animal experiments were conducted in accordance with Animal Research: Reporting of In Vivo Experiments guidelines (Percie du Sert et al., 2020) and the Institutional Animal Care and Use Committee of Central South University.

\section{Reagents and Antibodies Reagents}

Standard LPS (E. coli 0111:B4, Cat No. tlrl-eblps), ultrapure LPS (E. coli 0111:B4, Cat No. tlrl-3pelps), nigericin (Cat No. tlrl-nig), ATP (Cat No. tlrl-atpl), and MSU (Cat No. tlrl-msu) were purchased from InvivoGen (San Diego, CA, United States); the cell lysis buffer (CLB) (Cat No. 9803) was bought from Cell Signaling Technology (Danvers, MA, United States); the mouse immunoglobin IgG protein (Cat No. ab198772) was purchased from Abcam (Cambridge, CB2 0AX, United Kingdom); Protein A/G PLUS-Agarose (Cat No. sc-2003) was obtained from Santa Cruz (Santa Cruz, CA, United States); mouse IL-1 $\beta$ (Cat No. 88-7013), tumor necrosis factor- $\alpha$ (TNF- $\alpha$ ) (Cat No. 88-7324), interleukin-6 (IL-6) (Cat No. 88-701364), and a human IL-1 $\beta$ (Cat No. BMS22) ELISA kit was bought from Thermo Fisher (Waltham, MA United States); and the CellTiter-Glo ${ }^{\circledR}$ Luminescent Cell Viability Assay (Cat No. G7572) was from Promega.

\section{Antibodies}

Anti- $\beta$-actin $(1: 10,000$, BH10D10) was bought from Cell Signaling Technology (Danvers, MA, United States); AntiNLRP3 (1:1,000. Cryo-2) and Anti-ASC (1:1,000, AL177) were purchased from Adipogen (San Diego, CA, United States); AntiCaspase-1 (1:1,000, ab179515) and Anti-NEK7 (1:10,000 ab133514) were bought from Abcam (Cambridge, CB2 0AX, United Kingdom); Anti-IL-1 $\beta$ (1:000 AF-401-NA; RRID: AB_416684) was obtained from RD systems (Tustin, CA, United States); the DyLight 488-labeled secondary antibody (1: 50, A120-100D2) was purchased from InvivoGen (San Diego, CA, United States); and FITC anti-mouse/human CD11b (101216, 1:500 for flow cytometry) and APC antimouse Ly-6G (127614, 1:500 for flow cytometry) were from BioLegend.

\section{Cell Culture}

THP-1 cells were obtained from American Type Culture Collection (Manassas, VA). C57BL/6 mice were injected intraperitoneally with $3 \%$ thioglycolate before collecting primary peritoneal macrophages. Peritoneal lavage was performed to harvest exudate cells and seeded in 48-well (2-3 $\left.\times 10^{5}\right)$ or 6 -well $\left(2 \times 10^{6}\right)$ culture plates. After $2 \mathrm{~h}$, the nonadherent cells were removed; the adherent monolayer cells were peritoneal macrophages. Primary peritoneal macrophages and THP-1 cells were cultured in the RPMI-1640 medium supplemented with $10 \%$ fetal bovine serum, $100 \mathrm{U} / \mathrm{ml}$ penicillin, and $100 \mu \mathrm{g} / \mathrm{ml}$ streptomycin at $37 \mathrm{C}$ in a humidified incubator of $5 \% \mathrm{CO}_{2}$.

\section{Cell Viability Assay}

Peritoneal macrophages and THP-1 cells were seeded in 96-well $\left(4 \times 10^{4}\right)$ culture plates. After treatment with PL $(1,5,10,20$, and 40) for $30 \mathrm{~min}, 100 \mu \mathrm{L}$ of CellTiter-Glo ${ }^{\circledR}$ Reagent was added to each well. We incubated the plate at room temperature for $10 \mathrm{~min}$ and recorded luminescence.

\section{Inflammasome Activation}

As previously reported (Wang et al., 2021), for NLRP3 inflammasome activation, macrophages were primed with LPS (100 $\mathrm{ng} / \mathrm{ml}$ ) for $3 \mathrm{~h}$, followed by PL or DMSO for $30 \mathrm{~min}$ and stimuli as follows: $5 \mathrm{mM}$ ATP or $10 \mu \mathrm{M}$ nigericin for $1 \mathrm{~h}$ and $200 \mu \mathrm{g} / \mathrm{ml} \mathrm{MSU}$ for $6 \mathrm{~h}$; differentiated adherent THP-1 cells were induced by $100 \mathrm{nM}$ PMA (phorbol-12-myristate-13-acetate) for $3 \mathrm{~h}$ and then primed with LPS $(1 \mu \mathrm{g} / \mathrm{ml})$ for $3 \mathrm{~h}$, followed by NLRP3 inflammasome activation stimulation: $5 \mathrm{mM}$ ATP or $10 \mu \mathrm{M}$ nigericin for $1 \mathrm{~h}$ or $200 \mu \mathrm{g} / \mathrm{ml} \mathrm{MSU}$ for $6 \mathrm{~h}$.

\section{ASC Oligomerization}

C57BL/6 mice peritoneal macrophages were primed with LPS for $3 \mathrm{~h}$, treated with PL or DMSO for $30 \mathrm{~min}$, and stimulated with nigericin for $1 \mathrm{~h}$, and then, the cells were lysed with the Triton buffer [50 mM Tris- $\mathrm{HCl}$ ( $\mathrm{pH}$ 7.5), $150 \mathrm{mM} \mathrm{NaCl}, 0.5 \%$ Triton X100] mixed with $0.1 \mathrm{mM}$ phenylmethylsulfonyl fluoride (PMSF) and the EDTA-free protease inhibitor cocktail for $10 \mathrm{~min}$ on ice. Then, the cell lysates were centrifuged at $6000 \mathrm{~g}$ for $15 \mathrm{~min}$ on ice to collect the supernatant and to resuspend pellets in the $200 \mu \mathrm{L}$ Triton buffer after washing twice. $2 \mathrm{mM}$ disuccinimidyl suberate (DSS) was added into the resuspended pellets and crosslinked for $30 \mathrm{~min}$ at $37^{\circ} \mathrm{C}$. All samples were dissolved in the sodium dodecyl sulfate (SDS) loading buffer and heated to $100^{\circ} \mathrm{C}$ for $10 \mathrm{~min}$ for protein denaturation so as to prepare for Western blotting. 


\section{ASC Speck Formation}

C57BL/6 mice peritoneal macrophages were seeded on chamber slides overnight. Then, macrophages were primed with LPS for $3 \mathrm{~h}$ and treated with PL or DMSO for $30 \mathrm{~min}$ and stimulated with nigericin or ATP for $1 \mathrm{~h}$. After that, the cells were fixed in $4 \%$ paraformaldehyde (PFA) for $10 \mathrm{~min}$, permeabilized with $0.1 \%$ Triton X-100 for $10 \mathrm{~min}$, and blocked with 3\% BSA in PBS for $1 \mathrm{~h}$. Cells were then stained with Anti-ASC (1:200 at $4^{\circ} \mathrm{C}$ overnight) and the DyLight 488-labeled secondary antibody (1:50 at room temperature for $45 \mathrm{~min}$ ). Macrophage nuclei were dyed with DAPI. A fluorescence microscope (Nikon Ti2-U) was used to check these stained cells and ASC specks.

\section{Immunoprecipitation and Western Blot}

After indicated nigericin stimulation for $1 \mathrm{~h}$, mice peritoneal macrophages were lysed in an immunoprecipitation (IP) buffer mixed with PMSF and the cocktail. Then, these cell lysates were reacted to specific antibodies ASC or NEK7 and protein $\mathrm{G}$ plus-agarose overnight and washed four times with the IP buffer. Immunoprecipitates were eluted by boiling with $1 \%$ (w/ v) SDS loading buffer.

The supernatants (SN) were immunoprecipitated with NLRP3 antibodies for $12 \mathrm{~h}$ at $4^{\circ} \mathrm{C}$ and protein $\mathrm{A} / \mathrm{G}$ agarose for $2 \mathrm{~h}$. The immunoprecipitants were washed six times with the IP buffer and boiled with $1 \%(\mathrm{w} / \mathrm{v})$ SDS loading buffer for $10 \mathrm{~min}$ for immunoblot analysis.

For Western blot, stimulated macrophages were lysed with CLB (CST) supplemented with the cocktail and PMSF and subsequently centrifuged at $12,000 \mathrm{~g}$ at $4^{\circ} \mathrm{C}$ for $10 \mathrm{~min}$. Protein concentrations were detected with a bicinchoninic acid assay (Pierce). An equal content of extracts was separated by SDS-PAGE and transferred onto 0.22-mm PVDF membranes (Merck Millipore).

\section{SDD-AGE}

Western blot of the NLRP3 aggregate was analyzed following published protocols (Hou et al., 2011; Jiang et al., 2017). The procedure is briefly described as follows: mice peritoneal macrophages were lysed with the Triton X-100 lysis buffer, supplemented with PMSF and the cocktail, and then centrifuged at $12,000 \mathrm{~g}$ at $4^{\circ} \mathrm{C}$ for $5 \mathrm{~min}$. Next, the cell lysates were resuspended in a $5 \times$ sample buffer $(2.5 \times \mathrm{TBE}, 50 \%$ glycerol, $10 \%$ SDS, and $0.0025 \%$ bromophenol blue) and run onto vertical $1.5 \%$ agarose gel. After electrophoresis for $1 \mathrm{~h}$ at a constant voltage of $80 \mathrm{~V}$ at $4^{\circ} \mathrm{C}$ in the running buffer $(1 \times \mathrm{TBE}$ and $0.1 \%$ SDS), the proteins were transferred onto 0.22-mm PVDF membranes for $1 \mathrm{~h}$ for the following immunoblot.

\section{ELISA Assay for Cytokines}

Levels of IL-1 $\beta$, IL-6, and TNF- $\alpha$ obtained from cell culture after stimulations and mice blood serum were detected in quantitative ELISA kits (eBioscience), according to the manufacturer's instructions.

\section{LDH Release Assay}

Levels of LDH release in cells after stimulations were determined using an LDH Cytotoxicity Assay Kit bought from Beyotime (Shanghai, China), according to the manufacturer's instructions.

\section{In vivo Endotoxemia Model}

Wild-type C57BL/6 mice were pretreated with PL $(50 \mathrm{mg} / \mathrm{kg}$ or $100 \mathrm{mg} / \mathrm{kg}$ ) or an empty solvent (as an empty control) for $0.5 \mathrm{~h}$ and then injected intraperitoneally with LPS $(20 \mathrm{mg} / \mathrm{kg})$. After $8 \mathrm{~h}$, mice were sacrificed; the blood serum was collected by heart puncture to detect concentrations of IL- $1 \beta$, IL- 6 , and TNF- $\alpha$ by ELISA; and the lungs were harvested for histology analysis.

\section{MSU-Induced Peritonitis In Vivo}

Wild-type C57BL/6 mice were pretreated with PL (100 mg/kg) or an empty solvent (as an empty control) for $0.5 \mathrm{~h}$. Next, they were injected intraperitoneally with $1 \mathrm{mg}$ MSU (dissolved in $500 \mu \mathrm{L}$ PBS) for $6 \mathrm{~h}$. Peritoneal lavage was performed using $10 \mathrm{ml}$ icecold PBS to collect peritoneal exudate fluids and concentrated for ELISA analysis with an Amicon Ultra $10 \mathrm{~K}$ filter (UFC900308) from Millipore. Peritoneal cells were collected and analyzed by flow cytometry.

\section{Lung W/D Weight Ratio}

The severity of pulmonary edema was estimated by calculating the lung wet/dry (W/D) weight ratio. After sacrifice, the left lobe of the lung was excised, washed with phosphate-buffered saline (PBS), and weighed to gain the "wet" weight. The left lung was then placed in an oven for approximately $72 \mathrm{~h}$ at $65^{\circ} \mathrm{C}$ until there were no changes in the weight to obtain the "dry" weight.

\section{Histological Analysis}

After PBS perfusion to the cardiac, the lower right lobe of the lung was cut and fixed in $4 \%$ paraformaldehyde solution at room temperature for $24 \mathrm{~h}$. After regular dehydration for histological sections, these specimens were embedded with paraffin. Next, sections were cut and mounted on polysine adhesion glass slides for subsequent hematoxylin and eosin staining using standard procedures. Slides were examined under a Nikon ECL IPSE Ci biological microscope, and images were captured with a Nikon DS-U3 color digital camera.

\section{Statistical Analysis}

All values in our experiments are shown as the mean \pm SD. Statistical analysis was performed using GraphPad Prism 8.0 software. Unpaired Student's t test was used for comparison of two groups. When comparing more than two groups, ANOVA with the Bonferroni test was used. The statistical significance was set at $p<0.05$.

\section{RESULTS}

\section{Piperlongumine Inhibits NLRP3 Inflammasome Activation in Mouse Macrophages}

We first examined the cytotoxicity of PL $(1-40 \mu \mathrm{M})$ by cell viability and proved that the doses of PL were not cytotoxic (Figure 1A). To explore whether PL inhibits NLRP3 inflammasome, we treated LPS-primed mouse peritoneal macrophages with PL to exclude the effects of PL on the 


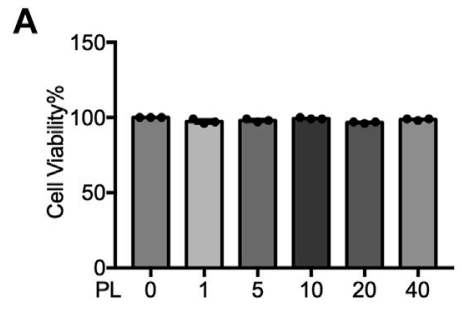

D

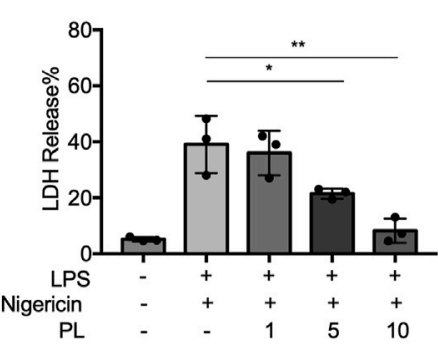

B

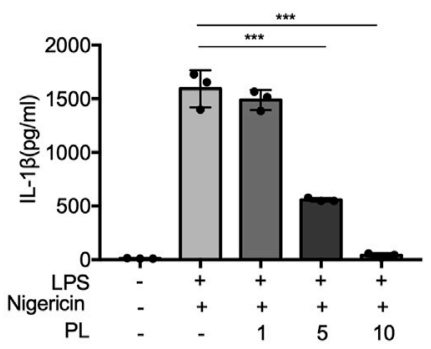

$\mathbf{E}$

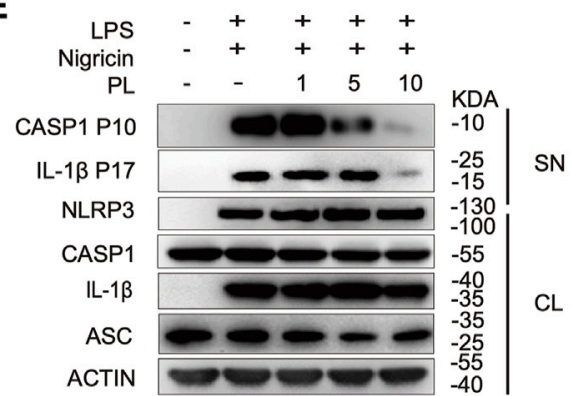

C

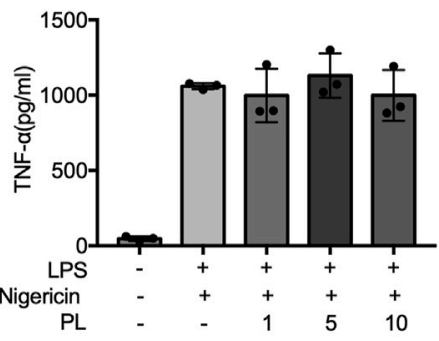

FIGURE 1 | PL dose-dependently inhibits nigericin-induced NLRP3 inflammasome activation. (A) Cell viability of PL (1-40 $\mu$ M) in peritoneal macrophages. (B-D) ELISA of IL-1 $\beta$ (B), TNF- $\alpha(\mathbf{C})$, and release of LDH (D) in supernatants from LPS-primed mouse peritoneal macrophages treated with $1-10 \mu M P L$ and stimulated with nigericin. (E) Immunoblot of supernatants or cell lysates from LPS-primed mouse peritoneal macrophages treated with 1-10 $\mu M$ PL and stimulated with nigericin. All data were representative of three independent experiments. Values shown are mean \pm SD. For statistical analysis, A-D were analyzed using one-way ANOVA and the Bonferroni test. ${ }^{*} p<0.05 ;{ }^{* *} p<0.01 ;{ }^{* \star *} p<0.001$.

priming signal and then added nigericin, an NLRP3 agonist by causing $\mathrm{K}+$ efflux. Interestingly, PL exhibited dose-dependent inhibitory effects on LPS + nigericin-induced IL- $1 \beta$ secretion and $\mathrm{LDH}$ release at the doses of $1-10 \mu \mathrm{M}$, while it had no effect on inflammasome-independent cytokine TNF- $\alpha$ production (Figures 1B-D). Similarly, the cleaved caspase-1 (p10) was reduced dose-dependently, measured by Western blot. Moreover, PL barely affected the expression of NLRP3, ASC, the precursors of IL-1 $\beta$, or the precursors of caspase- 1 (Figure 1E).

We further observed that PL inhibited IL-1 $\beta$ secretion, LDH release, and caspase- 1 cleavage when macrophages were treated with other NLRP3 agonists, including ATP and MSU (Figures 2A-D). Taken together, these results demonstrated the inhibitory effects of PL on the NLRP3 inflammasome in mouse macrophages.

\section{Piperlongumine Suppresses NLRP3 Inflammasome Activation in THP-1 Cells}

To further examine whether PL inhibits NLRP3 inflammasome in human cells, we detected the effects in THP-1 cells. First, we detected the cytotoxicity of PL $(1-40 \mu \mathrm{M})$ by cell viability and proved that the doses of PL were not cytotoxic (Figure 3A). Treating PL with PMA-primed THP-1 cells, we observed the declined IL-1 $\beta$ secretion and $\mathrm{LDH}$ release when challenged with nigericin, ATP, and MSU (Figures 3B-E). Thus, PL exerts an inhibitory role in NLRP3 inflammasome activation in human cells.

\section{Piperlongumine Interrupts ASC Speck Formation}

Next, we explored how PL inhibits NLRP3 activation. ASC speck formation is an essential step for NLRP3 activation (Oroz et al., 2016; Green et al., 2018), and then, we intended to determine whether PL has a regulatory role in ASC speck formation. With immunofluorescence microscopy analysis, we observed that PL markedly decreased the percentage of macrophages containing the ASC speck after stimulated with nigericin or ATP (Figures 4A,B). In common with the results of microscopy, PL distinctly reduced appearance of large multimeric ASC complexes in chemical cross-linking agents by Western blot (Figure 4C). Thus, the results indicated that PL blocks ASC oligomerization.

\section{Piperlongumine Inhibits NLRP3 Inflammasome Assembly}

Since ASC speck formation is a result of ASC recruitment to NLRP3 (Martinon et al., 2009; Davis et al., 2011), we next investigated whether PL influenced the interaction between them. By performing immunoprecipitation of ASC and NLRP3, we observed that PL markedly interrupted the ASC-NLRP3 association (Figure 5A), suggesting that $\mathrm{PL}$ targets the upstream of recruitment of ASC to NLRP3. Before recruiting ASC, NLRP3 first aggregates with the help of NEK7, a newly described component of NLRP3 inflammasome (He et al., 2016). We then detected the interaction between NEK7 and NLRP3. When treated with PL, the NEK7-NLRP3 association was disrupted (Figure 5B). Accordingly, the endogenous oligomerization of NLRP3 was 

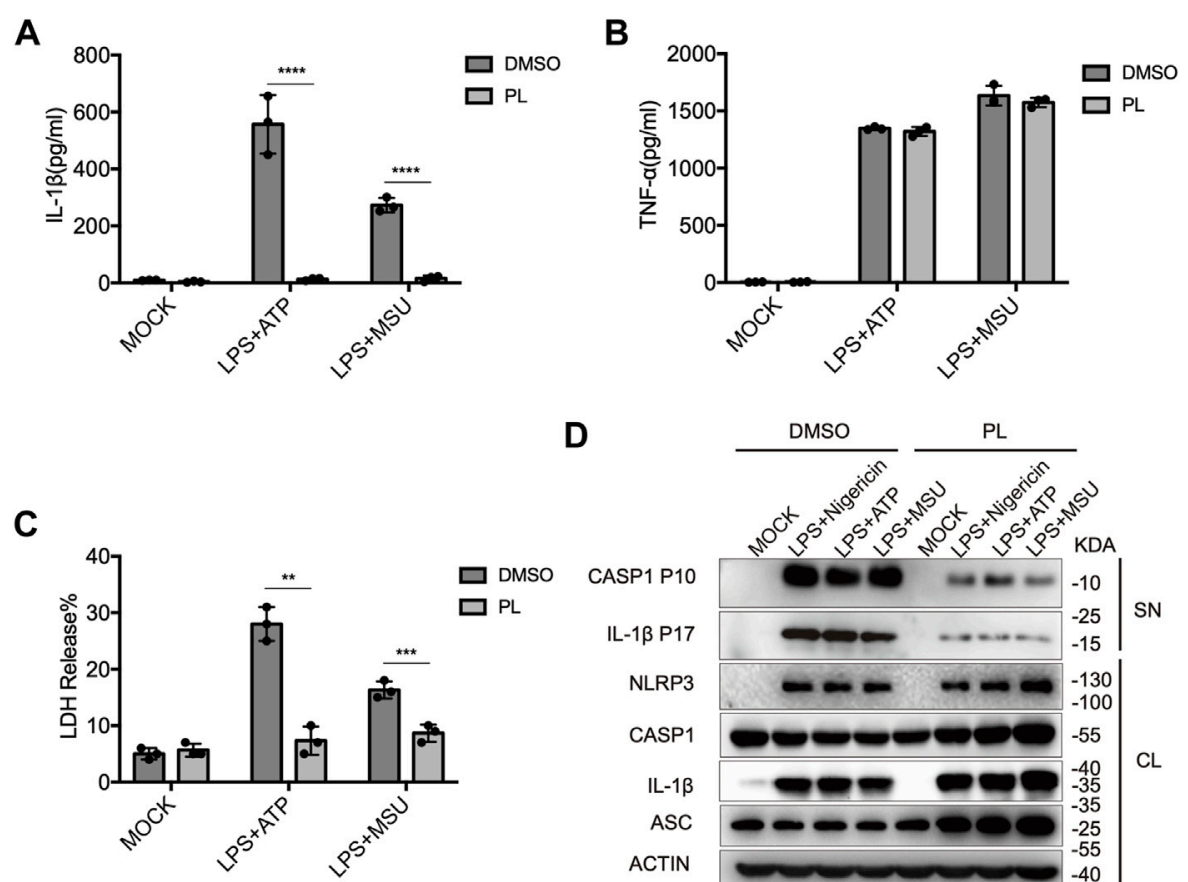

D

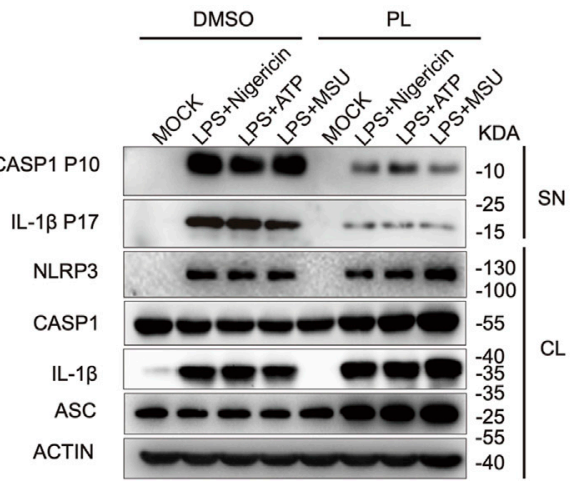

FIGURE 2 |PL inhibits ATP or MSU-induced NLRP3 inflammasome activation. (A-C) ELISA of IL-1 $\beta$ (A), TNF- $\alpha$ (B), and release of LDH (C) in supernatants from LPS-primed mouse peritoneal macrophages treated with $10 \mu \mathrm{M} \mathrm{PL}$ or DMSO and stimulated with ATP or MSU. (D) Immunoblot of supernatants or cell lysates from LPSprimed mouse peritoneal macrophages treated with $10 \mu \mathrm{M}$ PL and stimulated with indicated stimuli. All data were representative of three independent experiments. Values shown are mean \pm SD. For statistical analysis, A, B, and C were analyzed using two-way ANOVA and the Bonferroni test; ${ }^{* \star} p<0.01 ;{ }^{* \star *} p<0.001$; ${ }^{\star \star \star \star} p<0.0001$.
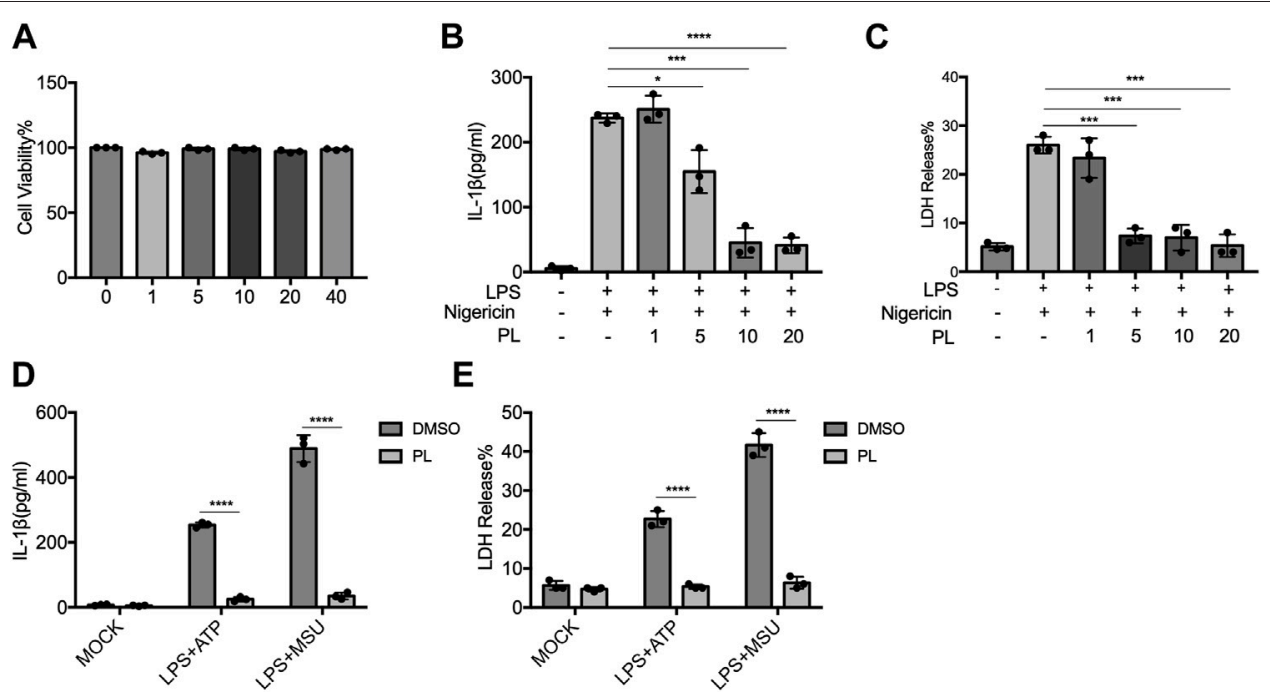

E

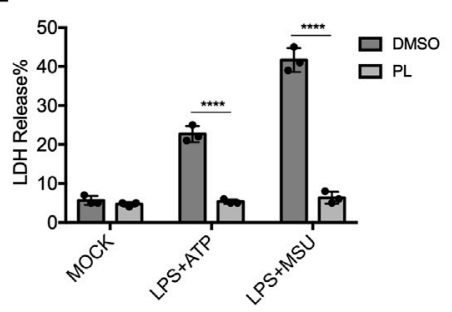

FIGURE 3 | PL blocks NLRP3 inflammasome activation in THP-1 cells. (A) Cell viability of PL (1-40 $\mu M$ ) in THP-1 cells. (B-C) ELISA of IL-1 3 (B) and release of LDH (C) in supernatants from PMA-primed THP-1 cells treated with 1-10 $\mu \mathrm{M}$ PL and challenged with nigericin. (D-E) ELISA of IL-1 $\beta$ (D) and release of LDH (E) in supernatants from PMA-primed THP-1 cells treated with $10 \mu \mathrm{M}$ PL or DMSO and stimulated with ATP or MSU. Values shown are mean \pm SD. For statistical analysis, A-C were analyzed using one-way ANOVA and the Bonferroni test. D and E were analyzed using two-way ANOVA and the Bonferroni test; ${ }^{\star} p<0.05 ;{ }^{\star \star \star} p<0.001$; ${ }^{\star \star \star \star} p<0.0001$. 

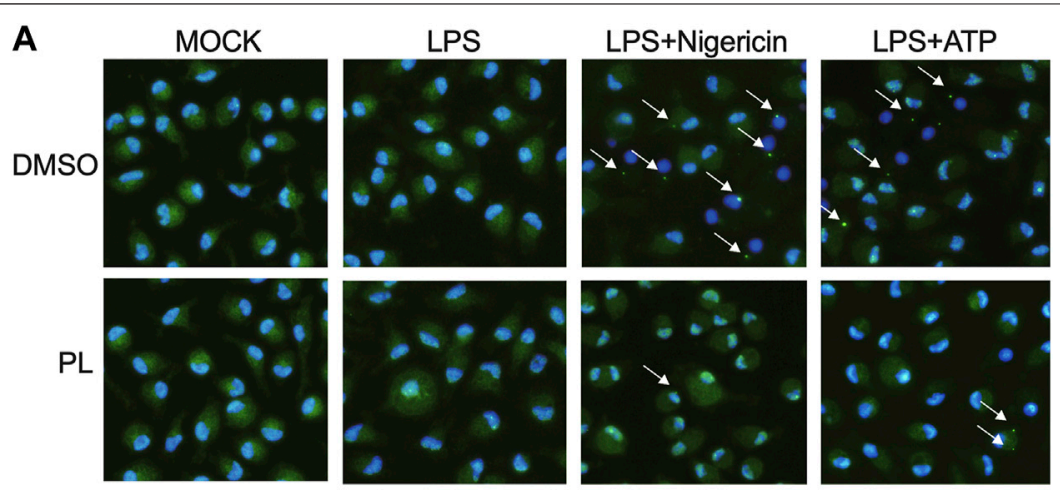

B
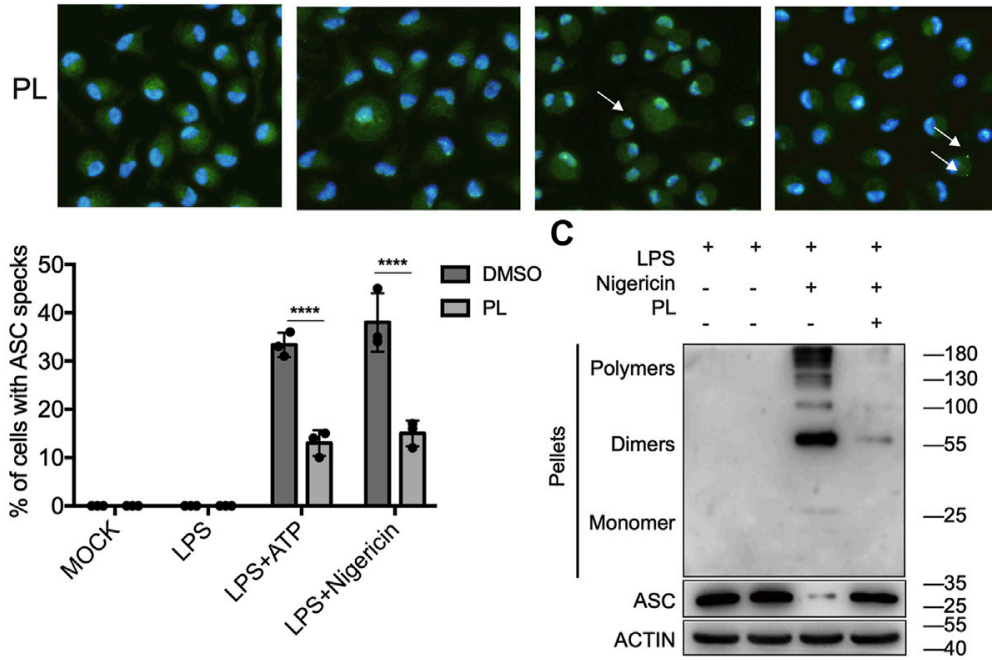

FIGURE 4 | PL suppresses ASC speck formation. (A, B) Immunofluorescence microscopy analysis of ASC specks in LPS-primed mouse peritoneal macrophages treated with $10 \mu \mathrm{M}$ PL or DMSO and stimulated with ATP or nigericin. (A) Representative images of ASC speck distribution in cells; ASC, green; nuclei, blue. White arrows indicate ASC specks. (B) Quantified percentage of cells containing an ASC speck. At least 100 peritoneal macrophages were collected for analysis. (C) Immunoblot analysis of ASC oligomerization in cross-linked cytosolic pellets of LPS-primed mouse peritoneal macrophages treated with $10 \mu \mathrm{M}$ PL and then stimulated with nigericin. Values shown are mean \pm SD. For statistical analysis, two-way ANOVA and the Bonferroni test were used; ${ }^{* \star \star \star} p<0.0001$. Data were collected from three independent experiments.

\section{A}

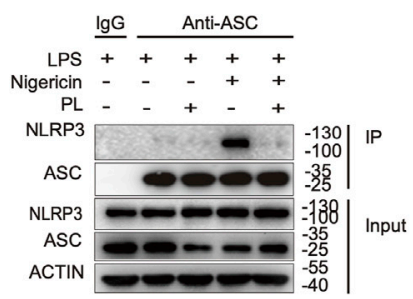

B

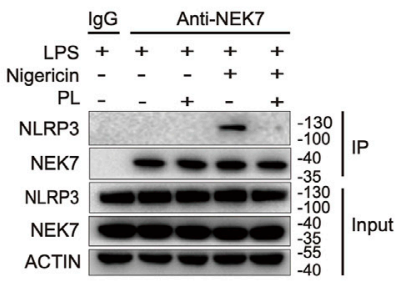

C

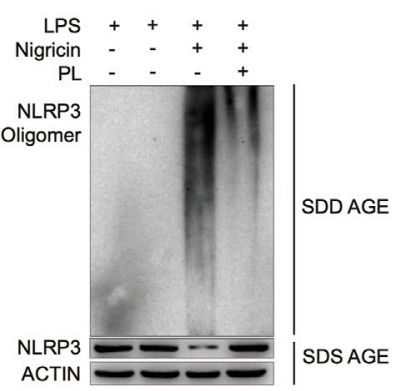

FIGURE 5 | PL interrupts NLRP3 inflammasome assembly. (A) Immunoblot analysis (immunoprecipitation) of the interaction between NLRP3 and ASC in LPS-primed mouse peritoneal macrophages treated with $10 \mu \mathrm{M}$ PL or DMSO and then stimulated with nigericin. (B) Immunoblot analysis (Immunoprecipitation) of the interaction between NEK7 and NLRP3 in LPS-primed primary macrophages treated with $10 \mu \mathrm{M} \mathrm{PL}$ or DMSO and then stimulated with nigericin. (C) Immunoblot analysis of NLRP3 oligomerization using SDD-AGE or SDS-PAGE assays in LPS-primed mouse peritoneal macrophages treated with $10 \mu \mathrm{M}$ PL or DMSO and then stimulated with nigericin.

dramatically decreased by using semi-denaturing detergent agarose gel electrophoresis (SDD-AGE) (Figure 5C). Thus, PL suppresses NLRP3 inflammasome activation through inhibiting the NLRP3 inflammasome assembly.

\section{Piperlongumine Suppresses NLRP3-Dependent Inflammation in vivo}

Finally, we investigated whether PL could inhibit NLRP3 inflammasome activation in vivo. Intraperitoneal injection of
LPS or MSU induces IL-1 $\beta$ secretion and neutrophil infiltration in a NLRP3-dependent manner (Martinon et al., 2006). Pretreatment of PL (50 mg/kg or $100 \mathrm{mg} / \mathrm{kg}$ ) could markedly attenuate release of IL- $1 \beta$ without affecting IL- 6 and TNF- $\alpha$ in serum induced by LPS injection (Figures 6A-C). Moreover, the PL-treated group showed moderate lung edema by calculating the W/D ratio (Figure 6D) and smaller bleeding spots, less inflammatory cell infiltration, and less impaired structures in lungs evaluated by histopathology, compared to the control group (Figure 6E). In another MSU-induced 


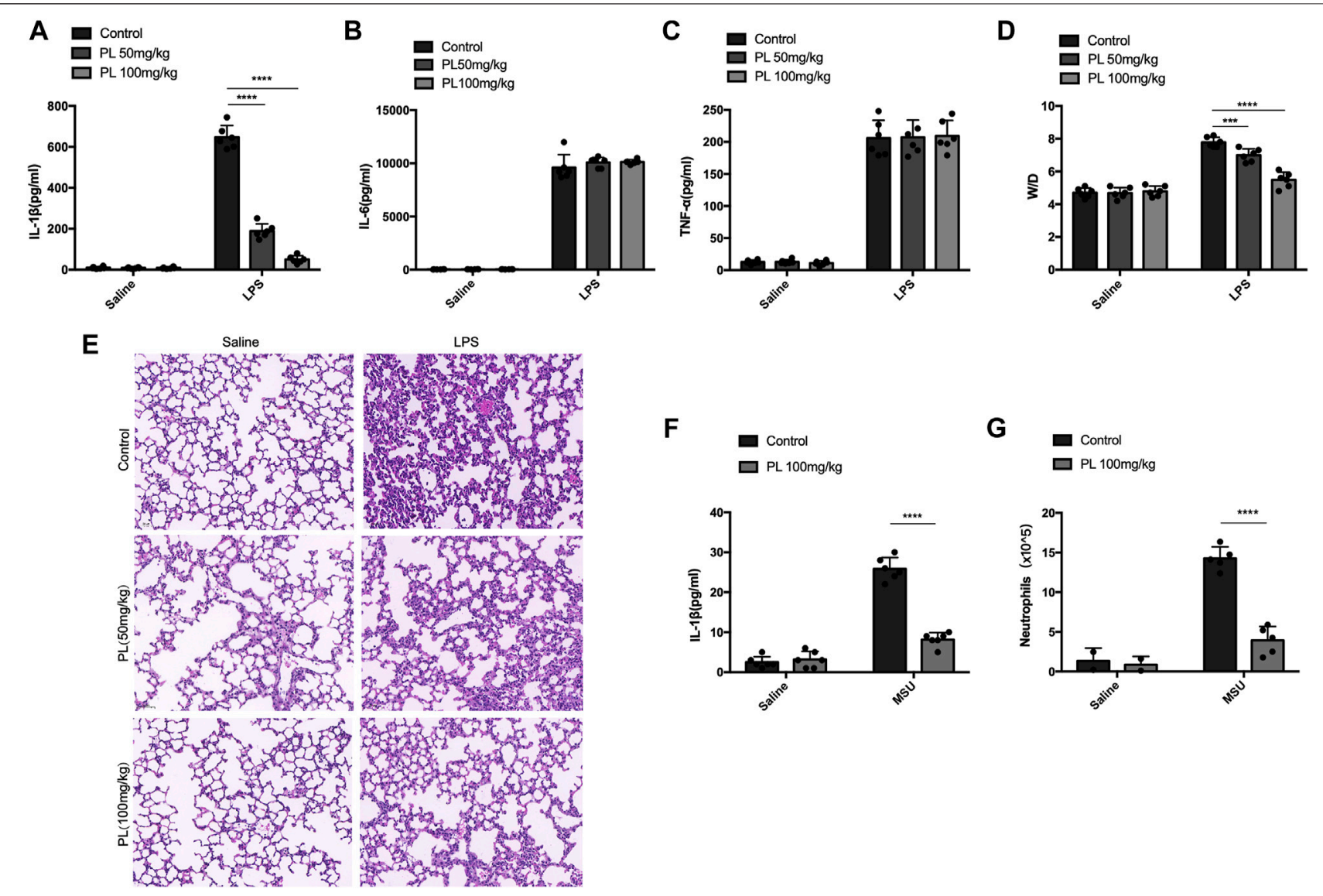

FIGURE 6 | PL disrupts NLRP3 inflammasome activation in vivo. (A-E) Effects of PL in endotoxemia. Wild-type mice with the C57BL/6 background were pretreated with intraperitoneal injection of PL (50 mg/kg or $100 \mathrm{mg} / \mathrm{kg}$ ) or N.S 30 min before intraperitoneal injection of LPS (20 mg/kg) for 8 h. ELISA analysis of IL-1 $\beta$ (A), IL-6 (B), and TNF- $\alpha$ (C) was performed in mice blood serum. (D) Lung W/D ratio of endotoxemic mice treated with PL (50 mg/kg or $100 \mathrm{mg} / \mathrm{kg}$ ). (E) Representative images of HE staining in lungs. (F, G) Effects of PL in MSU-induced peritonitis. Wild-type mice with the C57BL/6 background were intraperitoneally injected of PL $(100 \mathrm{mg} / \mathrm{kg}$ ) or N.S 30 min before intraperitoneal injection of MSU (1 mg) for 6 h. ELISA of IL-1 $\beta$ (F) and neutrophils (flow cytometry) (G) in the peritoneal cavity fluid were quantified. Values presented are mean \pm SD. Two-way ANOVA and the Bonferroni test were used for the statistical analysis; ${ }^{\star \star \star} p<0.001 ;{ }^{\star \star \star \star} p<0.0001$. Data were collected from three independent experiments.

peritonitis model, PL also exhibited inhibitory effects on NLRP3 inflammasome reflected by reduced IL-1 $\beta$ (Figure 6F) and recruitment of neutrophils (Figure 6G) in the lavage fluid. Taken together, these data proved that PL could inhibit NLRP3-dependent inflammation in vivo.

\section{DISCUSSION}

Piperlongumine, a kind of amid alkaloids, is an extract from the fruits of long pepper plants in Southern India and Southeast Asia. It not only flavors food tastes but also protects human health. Numerous studies have reported its anticancer function in different types of tumors both in vitro and in vivo, including colon, pancreatic, gastric, cholangio, lung, and prostate cancers (Randhawa et al., 2013; Dhillon et al., 2014; Ginzburg et al., 2014; Duan et al., 2016; Thongsom et al., 2017; Hałas-Wiśniewska et al., 2020). The anticancer properties of PL were demonstrated through cell cycle arrest, pro-apoptosis, anti-invasiveness, and antiangiogenesis by targeting JAK-STAT, NF-kB, or PI3K/ AKT/mTOR pathways (Farooqi et al., 2018; Piska et al., 2018). Recently, a few studies have uncovered the role of PL in alleviating sorts of inflammatory disorders, such as colitis, amyloidogenesis, liver fibrosis, diabetes, and psoriasis-like skin inflammation, suggesting an anti-inflammatory effect of PL (Gu et al., 2018; Chilvery et al., 2020; Thatikonda et al., 2020; Xu P. et al., 2021). In addition, these studies have proved that PL inhibits pro-inflammatory cytokine (TNF- $\alpha$ and IL-6) production mainly through suppressing the NF- $\mathrm{B}$ signal and iNOS expression. However, only this mechanism could not explain the role of PL under so many inflammatory conditions.

In this study, we demonstrated that PL is an inhibitor of NLRP3 inflammasome (Figure 7). Treated human or murine LPS-primed macrophages with PL could inhibit NLRP3 inflammasomeinduced IL- $1 \beta$ production and pyroptotic cell death without affecting inflammasome-independent cytokine TNF- $\alpha$ production. We noted that different from the previous study, PL did not suppress 


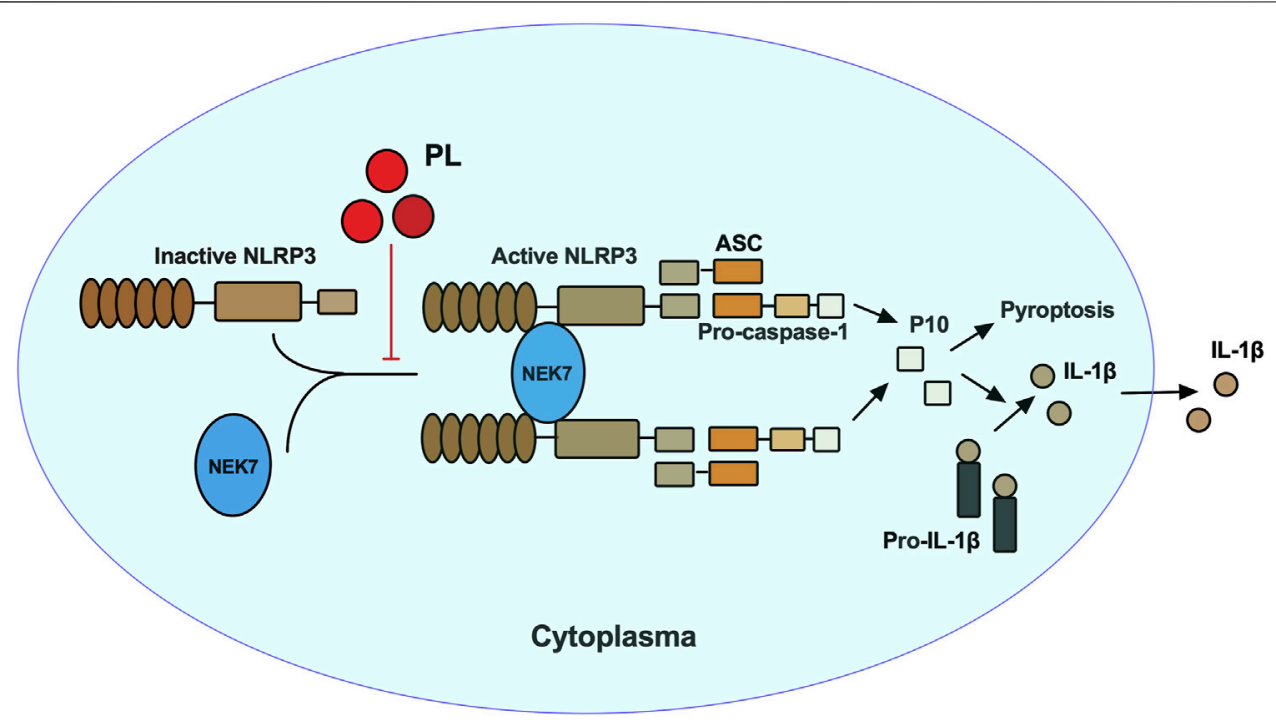

FIGURE 7 | Mechanism of PL inhibits NLRP3 inflammasome activation. PL blocks NLRP3 inflammasome activation by disrupting the interaction between NEK7 and NLRP3 and subsequent NLRP3 oligomerization.

TNF- $\alpha$ production in this model because of the fact that PL addition was after the NF- $\kappa \mathrm{B}$ signal activation. Moreover, it presented the specificity of PL in NLRP3 inflammasome without affecting the NF$\kappa \mathrm{B}$ signal. To further demonstrate whether PL could inhibit NLRP3 inflammasome in vivo, we first adopted an LPS-induced endotoxemia murine model and observed that PL markedly alleviated the inflammation, which is in line with a previous study (Lee et al., 2013). In another MSU-induced peritonitis model, PL exhibited similar effects by suppressing IL-1 $\beta$ production and neutrophil infiltration, both of which were dependent on NLRP3 inflammasome. Mechanistically, PL could inhibit the NLRP3 inflammasome assembly. By checking the ASC speck, an NLRP3 inflammation activation marker, we noticed that PL may target the upstream of ASC speck formation. Although performing SDD-AGE and immunoprecipitation, we demonstrated that PL interrupted NLRP3 oligomerization and the interaction between NLRP3 and NEK7, a newly recognized partner that bridges the bond of adjacent NLRP3 to form NLRP3 aggregates (He et al., 2016). However, the detailed molecular mechanism for PL that blocks the interaction between NLRP3 and NEK7 is not clear, which still remains further investigation.

A previous study treated macrophages with PL before LPS priming and found that PL could inhibit NLRP3 inflammasome activation through disruption of the NF- $\mathrm{BB}$ signaling pathway (Huang et al., 2021). However, in our study, we treated PL after LPS priming to exclude affecting the NF- $\kappa \mathrm{B}$ signal, and we found that PL could inhibit NLRP3 inflammasome activation through inhibiting the interaction of NLRP3 and NEK7 rather than the expression of NLRP3. Our study found a different anti-inflammatory mechanism of PL. Taken together, our study and previous study indicated that PL not only widely suppresses inflammatory response through the NF- $\kappa \mathrm{B}$ signaling pathway but also specifically inhibits NLRP3 inflammasome activation.
NLRP3 inflammasome is the most well-studied inflammasome. Numerous studies have indicated that excessive NLRP3 inflammasome activation is harmful to the host immune system and can lead to many diseases that are related to the long-term inflammatory process including type 2 diabetes mellitus, atherosclerosis, rheumatoid arthritis, and gout (Swanson et al., 2019). Disruption of NLRP3 inflammasome activation exhibits a therapeutic role to these diseases, thus apparently indicating its promising property in dealing with inflammatory related disorders. Accordingly, several compounds have been discovered for inhibiting NLRP3 inflammasome, and among them, MCC950 is the most wellstudied NLRP3 inhibitor. By directly interacting with NLRP3, MCC950 leads to an inactive NLRP3 conformation (TapiaAbellán et al., 2019). Besides MCC950, there are a serial of inhibitors directly interacting with NLRP3 and inhibiting NLRP3 ATPase activity, including CY-09, Bay 11-7082, OLT1177 dapansutrile, INF39, MNS, and BOT-4-one (Swanson et al., 2019). Apart from directly interacting with NLRP3, there are some inhibitors that control the NLRP3 activation in a posttranslational modification manner, such as SP600125, which disrupts the phosphorylation of NLRP3 and ASC; G5 prohibits the deubiquitination of NLRP3 (Jiang et al., 2020). In addition, some inhibitors target the association between NLRP3 and ASC, such as cardamonin (Jiang et al., 2020), SI-2 (Liu et al., 2020), and C646 (Xu X. et al., 2021), and some inhibitors block the interaction between NLRP3 and NEK7, including ordonin (Swanson et al., 2019) and ginsenoside Rg3 (Jiang et al., 2020). Our study added another natural inhibitor for NLRP3 by interrupting the interaction between NLRP3 and NEK7.

In summary, our study identified PL as an NLRP3 inhibitor by interrupting the assembly of the inflammasome, providing a new view of the anti-inflammatory mechanism of PL. Moreover, given 
aberrant activation of NLRP3 inflammasome leads to many inflammatory diseases; our study indicated the potential application of PL in NLRP3-related diseases.

\section{DATA AVAILABILITY STATEMENT}

The original contributions presented in the study are included in the article/Supplementary Material; further inquiries can be directed to the corresponding authors.

\section{ETHICS STATEMENT}

The animal study was reviewed and approved by Central South University.

\section{REFERENCES}

Bai, R., Lang, Y., Shao, J., Deng, Y., Refuhati, R., and Cui, L. (2021). The Role of NLRP3 Inflammasome in Cerebrovascular Diseases Pathology and Possible Therapeutic Targets. ASN Neuro 13, 17590914211018100. doi:10.1177/ 17590914211018100

Chen, W., Lian, W., Yuan, Y., and Li, M. (2019). The Synergistic Effects of Oxaliplatin and Piperlongumine on Colorectal Cancer Are Mediated by Oxidative Stress. Cel. Death Dis. 10, 600. doi:10.1038/s41419-019-1824-6

Chilvery, S., Bansod, S., Saifi, M. A., and Godugu, C. (2020). Piperlongumine Attenuates Bile Duct Ligation-Induced Liver Fibrosis in Mice via Inhibition of TGF- $\beta 1 /$ Smad and EMT Pathways. Int. Immunopharmacol. 88, 106909. doi:10.1016/j.intimp.2020.106909

Davis, B. K., Wen, H., and Ting, J. P. (2011). The Inflammasome NLRs in Immunity, Inflammation, and Associated Diseases. Annu. Rev. Immunol. 29, 707-735. doi:10.1146/annurev-immunol-031210-101405

Dhillon, H., Chikara, S., and Reindl, K. M. (2014). Piperlongumine Induces Pancreatic Cancer Cell Death by Enhancing Reactive Oxygen Species and DNA Damage. Toxicol. Rep. 1, 309-318. doi:10.1016/j.toxrep.2014.05.011

Duan, C., Zhang, B., Deng, C., Cao, Y., Zhou, F., Wu, L., et al. (2016). Piperlongumine Induces Gastric Cancer Cell Apoptosis and G2/M Cell Cycle Arrest Both In Vitro and In Vivo. Tumour Biol. 37, 10793-10804. doi:10.1007/s13277-016-4792-9

Duewell, P., Kono, H., Rayner, K. J., Sirois, C. M., Vladimer, G., Bauernfeind, F. G., et al. (2010). NLRP3 Inflammasomes Are Required for Atherogenesis and Activated by Cholesterol Crystals. Nature 464, 1357-1361. doi:10.1038/ nature 08938

Farooqi, A. A., Attar, R., Yaylim, I., Qureshi, M. Z., Todorovska, M., Karatoprak, G. Ş., et al. (2018). Piperlongumine as Anticancer Agent: The story So Far about Killing many Birds with One Stone. Cel. Mol. Biol. (Noisy-le-grand) 64, 102-107. doi: $10.14715 / \mathrm{cmb} / 2018.64 .11 .19$

Ginzburg, S., Golovine, K. V., Makhov, P. B., Uzzo, R. G., Kutikov, A., and Kolenko, V. M. (2014). Piperlongumine Inhibits NF- $\kappa B$ Activity and Attenuates Aggressive Growth Characteristics of Prostate Cancer Cells. Prostate 74, 177-186. doi:10.1002/pros.22739

Green, J. P., Yu, S., Martín-Sánchez, F., Pelegrin, P., Lopez-Castejon, G., Lawrence, C. B., et al. (2018). Chloride Regulates Dynamic NLRP3-dependent ASC Oligomerization and Inflammasome Priming. Proc. Natl. Acad. Sci. U S A. 115, E9371-e9380. doi:10.1073/pnas.1812744115

Gu, S. M., Lee, H. P., Ham, Y. W., Son, D. J., Kim, H. Y., Oh, K. W., et al. (2018). Piperlongumine Improves Lipopolysaccharide-Induced Amyloidogenesis by Suppressing NF-KappaB Pathway. Neuromolecular Med. 20, 312-327. doi:10.1007/s12017-018-8495-9

Hałas-Wiśniewska, M., Zielińska, W., Izdebska, M., and Grzanka, A. (2020). The Synergistic Effect of Piperlongumine and Sanguinarine on the Non-small Lung Cancer. Molecules 25, 3045. doi:10.3390/molecules 25133045

\section{AUTHOR CONTRIBUTIONS}

$\mathrm{XZ}$ and $\mathrm{RZ}$ were involved in project conceptualization. JS was involved in daily project operations. JS, YX, and HW collected data. JS and ZY were responsible for analytical procedures. All authors were involved in data analysis and interpretation and have reviewed and approved this manuscript.

\section{FUNDING}

This work was supported by the Major Science and Technology Project of Hainan Province (Grant No.ZDYF2020148), and funded by Hainan Province Clinical Medical Center, and the National Natural Science Foundation of China $(82102305,81960006)$.

He, Y., Zeng, M. Y., Yang, D., Motro, B., and Núñez, G. (2016). NEK7 Is an Essential Mediator of NLRP3 Activation Downstream of Potassium Efflux. Nature 530, 354-357. doi:10.1038/nature16959

Heneka, M. T., Kummer, M. P., Stutz, A., Delekate, A., Schwartz, S., Vieira-Saecker, A., et al. (2013). NLRP3 Is Activated in Alzheimer's Disease and Contributes to Pathology in APP/PS1 Mice. Nature 493, 674-678. doi:10.1038/nature11729

Hou, F., Sun, L., Zheng, H., Skaug, B., Jiang, Q. X., and Chen, Z. J. (2011). MAVS Forms Functional Prion-like Aggregates to Activate and Propagate Antiviral Innate Immune Response. Cell 146, 448-461. doi:10.1016/j.cell.2011.06.041

Huang, C. H., Wang, S. C., Chen, I. C., Chen, Y. T., Liu, P. L., Fang, S. H., et al. (2021). Protective Effect of Piplartine against LPS-Induced Sepsis through Attenuating the MAPKs/NF- $\kappa$ B Signaling Pathway and NLRP3 Inflammasome Activation. Pharmaceuticals (Basel) 14, 588. doi:10.3390/ph14060588

Jiang, H., He, H., Chen, Y., Huang, W., Cheng, J., Ye, J., et al. (2017). Identification of a Selective and Direct NLRP3 Inhibitor to Treat Inflammatory Disorders. J. Exp. Med. 214, 3219-3238. doi:10.1084/jem.20171419

Jiang, H., Gong, T., and Zhou, R. (2020). The Strategies of Targeting the NLRP3 Inflammasome to Treat Inflammatory Diseases. Adv. Immunol. 145, 55-93. doi:10.1016/bs.ai.2019.11.003

Kim, N., Do, J., Bae, J. S., Jin, H. K., Kim, J. H., Inn, K. S., et al. (2018). Piperlongumine Inhibits Neuroinflammation via Regulating NF- $\kappa B$ Signaling Pathways in Lipopolysaccharide-Stimulated BV2 Microglia Cells. J. Pharmacol. Sci. 137, 195-201. doi:10.1016/j.jphs.2018.06.004

Lee, W., Yoo, H., Kim, J. A., Lee, S., Jee, J. G., Lee, M. Y., et al. (2013). Barrier Protective Effects of Piperlonguminine in LPS-Induced Inflammation In Vitro and In Vivo. Food Chem. Toxicol. 58, 149-157. doi:10.1016/j.fct.2013.04.027

Liu, L., Xu, X., Zhang, N., Zhang, Y., and Zhao, K. (2020). Acetylase Inhibitor SI-2 Is a Potent Anti-inflammatory Agent by Inhibiting NLRP3 Inflammasome Activation. Int. Immunopharmacol. 87, 106829. doi:10.1016/ j.intimp.2020.106829

Lu, C., Zhang, B., Xu, T., Zhang, W., Bai, B., Xiao, Z., et al. (2019). Piperlongumine Reduces Ovalbumin-induced Asthma and Airway Inflammation by Regulating Nuclear factor-kB Activation. Int. J. Mol. Med. 44, 1855-1865. doi:10.3892/ ijmm.2019.4322

Mao, K., Chen, S., Chen, M., Ma, Y., Wang, Y., Huang, B., et al. (2013). Nitric Oxide Suppresses NLRP3 Inflammasome Activation and Protects against LPSInduced Septic Shock. Cell Res 23, 201-212. doi:10.1038/cr.2013.6

Martinon, F., Pétrilli, V., Mayor, A., Tardivel, A., and Tschopp, J. (2006). Goutassociated Uric Acid Crystals Activate the NALP3 Inflammasome. Nature 440, 237-241. doi:10.1038/nature04516

Martinon, F., Mayor, A., and Tschopp, J. (2009). The Inflammasomes: Guardians of the Body. Annu. Rev. Immunol. 27, 229-265. doi:10.1146/ annurev.immunol.021908.132715

Masters, S. L., Dunne, A., Subramanian, S. L., Hull, R. L., Tannahill, G. M., Sharp, F. A., et al. (2010). Activation of the NLRP3 Inflammasome by Islet Amyloid Polypeptide Provides a Mechanism for Enhanced IL-1 $\beta$ in Type 2 Diabetes. Nat. Immunol. 11, 897-904. doi:10.1038/ni.1935 
Oroz, J., Barrera-Vilarmau, S., Alfonso, C., Rivas, G., and De Alba, E. (2016). ASC Pyrin Domain Self-Associates and Binds NLRP3 Protein Using Equivalent Binding Interfaces. J. Biol. Chem. 291, 19487-19501. doi:10.1074/jbc.M116.741082

Percie du Sert, N., Hurst, V., Ahluwalia, A., Alam, S., Avey, M. T., Baker, M., et al. (2020). The ARRIVE Guidelines 2.0: Updated Guidelines for Reporting Animal Research. J. Cereb. Blood Flow Metab. 40, 1769-1777. doi:10.1177/ 0271678 X20943823

Piska, K., Gunia-Krzyżak, A., Koczurkiewicz, P., Wójcik-Pszczoła, K., and Pękala, E. (2018). Piperlongumine (Piplartine) as a lead Compound for Anticancer Agents - Synthesis and Properties of Analogues: A Mini-Review. Eur. J. Med. Chem. 156, 13-20. doi:10.1016/j.ejmech.2018.06.057

Randhawa, H., Kibble, K., Zeng, H., Moyer, M. P., and Reindl, K. M. (2013). Activation of ERK Signaling and Induction of colon Cancer Cell Death by Piperlongumine. Toxicol. Vitro 27, 1626-1633. doi:10.1016/j.tiv.2013.04.006

Rawat, L., Hegde, H., Hoti, S. L., and Nayak, V. (2020). Piperlongumine Induces ROS Mediated Cell Death and Synergizes Paclitaxel in Human Intestinal Cancer Cells. Biomed. Pharmacother. 128, 110243. doi:10.1016/ j.biopha.2020.110243

Shin, S. H., Lee, J. S., Zhang, J. M., Choi, S., Boskovic, Z. V., Zhao, R., et al. (2020). Synthetic Lethality by Targeting the RUVBL1/2-TTT Complex in mTORC1Hyperactive Cancer Cells. Sci. Adv. 6, eaay9131. doi:10.1126/sciadv.aay9131

Swanson, K. V., Deng, M., and Ting, J. P. (2019). The NLRP3 Inflammasome: Molecular Activation and Regulation to Therapeutics. Nat. Rev. Immunol. 19, 477-489. doi:10.1038/s41577-019-0165-0

Tapia-Abellán, A., Angosto-Bazarra, D., Martínez-Banaclocha, H., De TorreMinguela, C., Cerón-Carrasco, J. P., Pérez-Sánchez, H., et al. (2019). MCC950 Closes the Active Conformation of NLRP3 to an Inactive State. Nat. Chem. Biol. 15, 560-564. doi:10.1038/s41589-019-0278-6

Thatikonda, S., Pooladanda, V., Sigalapalli, D. K., and Godugu, C. (2020). Piperlongumine Regulates Epigenetic Modulation and Alleviates Psoriasislike Skin Inflammation via Inhibition of Hyperproliferation and Inflammation. Cel Death Dis 11, 21. doi:10.1038/s41419-019-2212-y

Thongsom, S., Suginta, W., Lee, K. J., Choe, H., and Talabnin, C. (2017). Piperlongumine Induces G2/M Phase Arrest and Apoptosis in
Cholangiocarcinoma Cells through the ROS-JNK-ERK Signaling Pathway. Apoptosis 22, 1473-1484. doi:10.1007/s10495-017-1422-y

Wang, Y. H., Morris-Natschke, S. L., Yang, J., Niu, H. M., Long, C. L., and Lee, K. H. (2014). Anticancer Principles from Medicinal Piper ( Hú Jiāo) Plants. J. Tradit Complement. Med. 4, 8-16. doi:10.4103/2225-4110.124811

Wang, D., Zhang, Y., Xu, X., Wu, J., Peng, Y., Li, J., et al. (2021). YAP Promotes the Activation of NLRP3 Inflammasome via Blocking K27-Linked Polyubiquitination of NLRP3. Nat. Commun. 12, 2674. doi:10.1038/s41467021-22987-3

Xu, P., Xiao, J., and Chi, S. (2021a). Piperlongumine Attenuates Oxidative Stress, Inflammatory, and Apoptosis through Modulating the GLUT-2/4 and AKT Signaling Pathway in Streptozotocin-Induced Diabetic Rats. J. Biochem. Mol. Toxicol. 35, 1-12. doi:10.1002/jbt.22763

Xu, X., Li, J., Long, X., Tao, S., Yu, X., Ruan, X., et al. (2021b). C646 Protects against DSS-Induced Colitis Model by Targeting NLRP3 Inflammasome. Front. Pharmacol. 12, 707610. doi:10.3389/fphar.2021.707610

Conflict of Interest: The authors declare that the research was conducted in the absence of any commercial or financial relationships that could be construed as a potential conflict of interest.

Publisher's Note: All claims expressed in this article are solely those of the authors and do not necessarily represent those of their affiliated organizations or those of the publisher, the editors, and the reviewers. Any product that may be evaluated in this article or claim that may be made by its manufacturer is not guaranteed or endorsed by the publisher.

Copyright (c) 2022 Shi, Xia, Wang, Yi, Zhang and Zhang. This is an open-access article distributed under the terms of the Creative Commons Attribution License (CC $B Y)$. The use, distribution or reproduction in other forums is permitted, provided the original author(s) and the copyright owner(s) are credited and that the original publication in this journal is cited, in accordance with accepted academic practice. No use, distribution or reproduction is permitted which does not comply with these terms. 